\title{
EDITORIAL
}

\section{Visibilidad científica: Compromiso y desafío para los investigadores de la Asociación Latinoamericana de la Papa}

\author{
J. Gabriel ${ }^{1 / *}$
}

Recibido: $30 / 11 / 2018$

Aceptado: $28 / 12 / 2018$

Accesible en línea: Diciembre 2018

\section{Introducción}

La investigación es una de las actividades más importantes de toda institución de que desarrolla conocimientos y tecnología la misma debe se plasmada en publicaciones científicas (Turpo y Medina 2013). Es claro que una investigación sin publicación no es investigación, o como declara el dicho "La ciencia que no se ve, no existe" (Turpo y Medina 2013). Más aún, en el entorno institucional en que se vive, es necesario facilitar la interacción entre la sociedad del conocimiento (Pacheco y Hurtado 2012) y los investigadores, lo que evita no solo gastos de mayores recursos, sino también la duplicidad de las investigaciones. En este contexto se debe comprender lo que significa visibilidad de la producción científica. Asimismo, para fines de posicionamiento en los rankings internacionales, es necesario conocer el impacto citacional de una investigación, lo que se logra gracias a la indexación (Turbo y Medina 2013).

\section{La importancia de la visibilidad científica}

La visibilidad científica está relacionada con la cantidad de materiales y publicaciones que produce una universidad, un centro de investigación o las sociedades científicas y que pueden ser vistas por la comunidad científica (Turpo y Medina 2013). Para lograr la mayor visibilidad científica, es necesario conocer los factores involucrados en el campo de la medición de la calidad científica (Ardito 2012). Esta visibilidad se puede lograr publicando una mayor cantidad de libros; creando una revista científica; adecuando las revistas de acuerdo a los criterios de las bases de datos; creando políticas institucionales de incentivos para la publicación de manuscritos; capacitando a los investigadores en todos los procesos de publicación. Sea cual fuere el camino a tomar, se deben seguir políticas serias para incrementar esta visibilidad.

Una revista científica es una publicación que cuenta con una periodicidad establecida, con artículos originales e inéditos, producto del resultado de investigaciones que son debidamente arbitrados por un comité editorial reconocido a nivel nacional e internacional y que, además, se apega estrictamente a la normativa establecida para las publicaciones, cuyo principal objetivo es comunicar ciencia" (Rojas y Rivera 2012). Por lo tanto el impacto citacional representa el número de veces que es citado el artículo por otros investigadores (Pacheco y Hurtado 2012, Turpo y Medina 2013).

Mencionar que la indexación es la medida natural de la producción científica de calidad, permite no solo que la comunidad regional se entere de la investigación local, sino además permiten a los académicos e investigadores de la región puedan llegar a comunidades locales, nacionales y, gracias al Open Access, a todo el mundo (Turpo y Medina 2013). Por ello la Vinculado a esta reunión, el CIP y la Asociación Europea de Investigación de la Papa (EAPR)

\footnotetext{
*Autor para correspondencia. E-mail: julio.gabriel@unesum.edu.ec, j.gabriel@proinpa.org

${ }^{1}$ Universidad Estatal del Sur de Manabì, Jipijapa, Manabì, Ecuador.
} 
importancia para las universidades e institutos de investigación de contar con revistas indexadas, y lograr que sus docentes e investigadores publiquen en revistas científicas de alto impacto. Asimismo, se debe implementar prácticas en la cual los investigadores citen artículos de las revistas científicas institucionales, y entre ellas se colaboren a elevar el nivel de visibilidad.

Es conveniente aclarar que el concepto de indización está relacionado a la técnica de análisis de documentos, el cual tiene por objetivo representar y describir el contenido de los mismos, mediante conceptos principales contenidos en ellos (palabras clave) o vocabularios controlados (descriptores, términos o encabezamientos de materia), con la finalidad de guiar al usuario en la recuperación de documentos a los que necesita acceder; al mismo tiempo se debe hacer diferencia la palabra indización de la indexación, que es otro término muy frecuentemente utilizado, mismo que se entiende como el proceso por el cual el buscador va rastreando el sitio en cuestión y al mismo tiempo va incorporando a su base de datos el contenido de esas URLs, es decir, se registra ordenadamente información para elaborar un índice (Turpo y Medina 2013). Este procedimiento es de vital importancia para las páginas en línea o web, ya que es el que permite que la página web aparezca en alguno de los buscadores más importantes, aquella página que no se haya indexado como debería no aparecerá entre los resultados de una búsqueda, en palabras sencillas indexar es agregar una página sin importar en que formato se encuentre a la lista de resultados de un buscador (Aguirre 2012). La tabla 1, muestra las principales indexadoras a nivel regional y del mundo, en las cuales las revistas científicas deben estar indexadas.

Tabla 1. Principales indexadoras o repositorios virtuales de artículos científicos.

\begin{tabular}{|c|c|c|c|}
\hline índice & Descripción & $\begin{array}{l}\text { Artículos } \\
\text { de texto } \\
\text { completo }\end{array}$ & $\begin{array}{l}\text { Tipo de } \\
\text { acceso a } \\
\quad \text { los } \\
\text { artículos }\end{array}$ \\
\hline Dialnet & $\begin{array}{l}\text { Repositorio o biblioteca virtual y base de datos especializada, creada en el año } \\
2001 \text { por la Universidad de La Rioja, España. Con predominio en Ciencias } \\
\text { Sociales y Humanidades, incluye también revistas de Ciencia y Tecnología. } \\
\text { Su objetivo es aumentar la difusión, visibilidad y accesibilidad de la literatura } \\
\text { científica hispana, potenciando el acceso libre y gratuito. Para conseguir ese } \\
\text { objetivo, Dialnet ofrece a los servicios de publicaciones de entidades cuya } \\
\text { actividad no tenga ánimo de lucro, el alojamiento de los textos completos de } \\
\text { sus publicaciones, tanto revistas como tesis doctorales y libros colectivos. La } \\
\text { Revista de la ALAP está en esta en este repositorio. }\end{array}$ & No & Gratuito \\
\hline Latindex & $\begin{array}{l}\text { Latindex creado en } 1997 \text { es conformado por } 19 \text { organismos } \\
\text { gubernamentales y académicos representantes de distintos países } \\
\text { latinoamericanos. Es un directorio que recoge información sobre revistas } \\
\text { científicas, profesionales y técnicas. En el } 2002 \text { se creó el Catálogo, que } \\
\text { contiene una selección de las revistas incluidas en el directorio de acuerdo a } \\
36 \text { criterios de calidad editorial internacional. El Catálogo Latindex no indiza } \\
\text { artículo por artículo, sino que entrega información descriptiva sobre la revista } \\
\text { a nivel de título. La Revista ALAP está en Directorio, pero se está } \\
\text { gestionando para ser incluida en Catálogo. }\end{array}$ & No & Gratuito \\
\hline $\begin{array}{c}\text { Clase (Citas } \\
\text { latinoamericanas } \\
\text { en Ciencias } \\
\text { Sociales y } \\
\text { Humanidades) }\end{array}$ & $\begin{array}{l}\text { CLASE y PERIÓDICA indizan revistas académicas de investigación, técnico- } \\
\text { profesionales y de difusión científica o cultural, editadas en países de América } \\
\text { Latina y el Caribe. También se incluyen revistas editadas por organismos } \\
\text { internacionales de alcance panamericano. Las revistas pueden ser } \\
\text { especializadas o multidisciplinarias, en formato impreso o electrónico. Las } \\
\text { revistas especializadas en ciencias sociales y humanidades se indizan en } \\
\text { CLASE, mientras que las de ciencia y tecnología se incluyen en PERIÓDICA. } \\
\text { Las multidisciplinarias pueden incluirse en alguna de las dos bases de datos, } \\
\text { conforme a la opinión del Comité de Selección. La Revista de la ALAP está } \\
\text { indizada. }\end{array}$ & $\mathrm{Si}$ & Gratuito \\
\hline
\end{tabular}


Continuación Tabla 1...

\begin{tabular}{|c|c|c|c|}
\hline índice & Descripción & $\begin{array}{c}\text { Artículos } \\
\text { de texto } \\
\text { completo }\end{array}$ & $\begin{array}{l}\text { Tipo de } \\
\text { acceso a } \\
\text { los } \\
\text { artículo } \\
\quad \mathrm{s}\end{array}$ \\
\hline $\begin{array}{c}\text { AJ (Directory } \\
\text { of open acces } \\
\text { journals) }\end{array}$ & $\begin{array}{l}\text { Directorio administrado y parcialmente financiado por el sistema de } \\
\text { Biblioteca de la Universidad de Lund. Indexa solo revistas en línea de } \\
\text { contenidos abiertos. Dentro de sus requisitos está que los contenidos sean } \\
\text { totalmente accesibles, no acepta embargos por períodos. Las revistas } \\
\text { seleccionadas son principalmente académicas y científicas, pone como } \\
\text { requisito que estén primariamente orientadas a un público conformado por } \\
\text { investigadores. No limita la postulación de revistas por idioma o área } \\
\text { temática. Su objetivo es incrementar la visibilidad y la facilidad de uso de las } \\
\text { revistas. La Revista ALAP no está en este directorio. }\end{array}$ & $\mathrm{Si}$ & Gratuito \\
\hline $\begin{array}{l}\text { Redalyc (Red } \\
\text { de revistas } \\
\text { científicas } \\
\text { de América } \\
\text { Latina y El } \\
\text { Caribe, } \\
\text { España y } \\
\text { Portugal) }\end{array}$ & $\begin{array}{l}\text { Creado en el } 2003 \text { por la Universidad Autónoma del Estado de México. } \\
\text { Indiza revistas principalmente de América Latina, España y Portugal. Tiene } \\
\text { una amplia cobertura de las ciencias sociales. Las postulaciones al índice son } \\
\text { en febrero y julio de cada año. Observa } 39 \text { criterios de selección, de los } \\
\text { cuales } 27 \text { son generales, } 12 \text { son básicos y } 5 \text { esenciales. Para poder ser } \\
\text { indizada, una revista debe cumplir al menos } 20 \text { de los } 27 \text { criterios. Si una } \\
\text { revista tiene versión impresa y digital, se sugiere postular solo una de las } \\
\text { versiones. La revista de la ALAP no está indizada. }\end{array}$ & $\mathrm{Si}$ & Gratuito \\
\hline $\begin{array}{c}\text { SCIELO } \\
\text { (Scientific } \\
\text { Electronic } \\
\text { Library Online) }\end{array}$ & $\begin{array}{l}\text { Creado por la Fundación de Apoyo a la Investigación del Estado de São } \\
\text { Paulo y por Centro Latinoamericano y del Caribe de Información en Ciencias } \\
\text { de la Salud. Esta biblioteca electrónica que incluye una colección } \\
\text { seleccionada de revistas científicas. Para su aceptación las revistas son } \\
\text { evaluadas según } 17 \text { criterios centrados en la calidad de los artículos } \\
\text { publicados, que deben ser originales y en su mayoría de corte científico y en } \\
\text { la implementación de un proceso de revisión por pares hecho por } \\
\text { especialistas. Las revistas que ingresen deben seguir rigurosamente la } \\
\text { metodología Scielo, así como el uso de metadatos y de referencias por } \\
\text { separado. Las revistas son aceptadas de forma inmediata si están incluidas en } \\
\text { Thompson Reuters (ex ISI), MEDLINE o PsycINFO. La Revista ALAP no } \\
\text { está indizada. }\end{array}$ & & \\
\hline SCOPUS & $\begin{array}{l}\text { Creado el año } 2004 \text { por la empresa Elsevier, esta base de datos cubre más de } \\
27000 \text { revistas. Cuenta con } 420 \text { títulos latinoamericanos y al menos un } 21 \% \\
\text { de los títulos cubiertos en Scopus están en idiomas diferentes al inglés. Para } \\
\text { evaluar la inclusión de nuevas revistas observa } 16 \text { criterios, agrupados en } 5 \\
\text { categorías: política editorial (incluye revisión por pares modalidad doble } \\
\text { ciego y diversidad de autores y de comité editorial), contenido (académico y } \\
\text { relevante al área de la revista), provisión de referencias bibliográficas y citas } \\
\text { correctas y bien estructuradas; y periodicidad. Es un indicador bibliométrico } \\
\text { en que se añaden títulos nuevos a Scopus dos veces al año: enero (para los } \\
\text { títulos enviados antes del } 1 \text { de setiembre del año anterior) y julio (para los } \\
\text { títulos enviados antes del } 1 \text { de marzo del presente año). La revista ALAP no } \\
\text { está en esta base de datos. }\end{array}$ & No & $\begin{array}{l}\text { Suscripci } \\
\text { ón }\end{array}$ \\
\hline $\begin{array}{l}\text { Web of Science } \\
\text { y Web of } \\
\text { nowledge: } \\
\text { ISI (Institute } \\
\text { of Scientific } \\
\text { Information) }\end{array}$ & $\begin{array}{l}\text { Fue adquirido el año } 2002 \text { por Thompson Reuters. Esta empresa es la } \\
\text { encargada de elaborar los índices Web of Science. Contiene las revistas } \\
\text { científicas consideradas más prestigiosas por su actor de impacto a nivel } \\
\text { global, y Web of Knowledge. Además de indizar revistas de ciencia también } \\
\text { incorpora títulos de ciencias sociales, artes y humanidades. La selección de } \\
\text { revistas está basada en la periodicidad; en que la revista publique } \\
\text { información bibliográfica en inglés; revisión por pares y tener un formato } \\
\text { que asegure la correcta citación de los artículos publicados. Este índice se ha } \\
\text { convertido en el indicador más reconocido de calidad editorial de las revistas } \\
\text { y de bibliometría. La Revista ALAP no está indizada en esta base. }\end{array}$ & No & $\begin{array}{c}\text { Suscripci } \\
\text { ón }\end{array}$ \\
\hline
\end{tabular}

Fuente: Turpo y Medina (2013). Producción intelectual y visibilidad científica. Apuntes Universitarios. Adaptado 
Por otro lado, si un investigador desea publicar en una revista internacional que se encuentre en la Web Knowledge o en el catálogo de SCI de la editorial ELSEVIER, debe hacerlo casi exclusivamente en inglés, formar parte de redes internacionales y pagar, en algunos casos, montos considerables para que pueda ser recibida (no necesariamente publicada), eso sin contar el sesgo de estas revistas hacia los países en desarrollo.

\section{Revista Latinoamericana de la Papa}

Esta publicación de la Asocoaciòn Latinomaricana de la Papa (ALAP), está dirigida a la comunidad científica de todas las ciencias relacionadas con la papa. Tiene una frecuencia semestral (Junio y Diciembre). Se aceptan artículos originales e inéditos en biotecnología, genómica, fisiología, nutrición y fertilización de cultivos, genética y fitomejoramiento, entomología, fitopatología, fitoprotección integrada, agroecología, malherbología, geomática, suelos, aguas y riego, poscosecha y agro-industrialización, desarrollo rural y agroempresarial, economía agrícola y mercadeo de productos agrícolas (Gabriel et al. 2018).

La Revista Latinoamericana de la Papa publicó 25 números desde el año 1988. Inicialmente publicó en versión impresa, logrando sacar un número cada año, hasta el año 2010. A partir del año 2011 a la fecha se publica digitalmente dos números por año, la primera en junio y segunda en Diciembre.

Por la directiva y el comité editorial de la Revista han pasado investigadores de reconocida trayectoria profesional de Perú, Argentina, Chile, Colombia, España, Ecuador, Bolivia, Costa Rica, Uruguay, USA, etc.

La Revista fue indizada en Latindex, Agris (FAO), OJS (Bolivia), UCBConocimiento (Bolivia), REDIB, PERIODICA (México), BIBLAT (México), DIALNET (España) y recientemente en EBSCO.

La Revista publicó un total de 215 publicaciones, de los cuales 12 fueron artículos invitados, 170 artículos científicos, 25 comunicaciones cortas y 8 revisiones. Del total de publicaciones, $29 \%$ corresponden a Bolivia, $23 \%$ a Perú, $13 \%$ a Argentina, $8 \%$ a Colombia, $8 \%$ a Ecuador, 4\% a Venezuela, 3\% a México y 4\% a Brasil. Países como España, Costa Rica, Honduras, Panamá, Nicaragua, Chile, USA y Dinamarca han contribuido con 1\% por país (Gabriel et al. 2018).

Los principales temas de publicación fueron: $31 \%$ en Fitopatología, 16\% en Entomología, 49\% en Fitomejoramiento, 31\% en Agronomía y Fisiología, 15\% en agroindustria, 13\% en RRGG, $11 \%$ en Metodologías Participativas y Economía, 9\% en Producción de semilla, 9\% temas institucionales, $6 \%$ en Biotecnología y $5 \%$ en Microbiología.

\section{Desafíos futuros}

Mantenerse vigente con la información científica, es muy compleja para una revista modesta como la nuestra. La Revista ALAP en la actualidad ya cuenta con su propio OJS (http://ojs.papaslatinas.org/index.php/rev-alap), la misma está en proceso de mejora continua. Se gestionó la indización a Scielo, REDALYC y DOAJ, sin éxito. Se está gestionando con las indexadoras mencionadas para que nuestra revista sea considerada nuevamente. Si logramos indexarnos será señal de que podemos acceder a indexarnos en otras bases como: Thomson, Scopus, SRI, Springer, etc. Sin embargo, todo depende del equipo de talentos humanos comprometidos y empoderados, la gestión de la información científica y de la calidad de la información científica que generemos y reportemos. 


\section{Referencias}

Aguirre, J. (2012). Respecto a las Indizaciones e Indexaciones. Revista Científica Ciencia Médica. 15. 1

Ardito, F. (2012). Seminario de Publicación Científica. Asamblea Nacional de Rectores. Pucallpa: Universidad Nacional de Ucayali.

Gabriel, J.; Huarte, M.; Salas, E. (2018). Visibility: The challenge of the Latin American Potato Journal. Revista Latinoamericana de la Papa 22 (2): 66 - 69.

Pacheco, J., \& Hurtado, A. (2012). Estrategias para mejorar el impacto de las publicaciones indexadas en Publindex, ISI, Scopues y SciELO: el caso de la Universidad Santo Tomás, Colombia. Revista Hallazgos, 19, 175-191.

Rojas, A., Rivera, S. (2012). Guía de buenas prácticas para revistas académicas en acceso abierto. ONG Derechos digitales. Disponible en: http://www.derechosdigitales.org SIR (2013).

Turpo, J.E.; Medina, G.E. (2013). Producción intelectual y visibilidad científica. Apuntes Universitarios. Revista de Investigación 2 (3): 9-18. 